\title{
Log laws or power laws: The scaling in the overlap region
}

\author{
M.V. Zagarola \\ Creare Inc., P.O. Box 71, Hanover, NH 03755 \\ A.E. Perry \\ Department of Mechanical and Manufacturing Engineering \\ Melbourne University, Parkville, Victoria 3052, Australia \\ and \\ A.J. Smits \\ Department of Mechanical and Aerospace Engineering \\ Princeton University, Princeton, N.J. 08544-0710
}

March 12, 1997

\begin{abstract}
The scaling in the overlap region of turbulent wall-bounded flows has long been the source of controversy, and until recently this controversy could not be addressed because measurements did not span a sufficient range of Reynolds number. Mean velocity surveys performed in a new pipe flow experiment span a very large range of Reynolds numbers, $31 \times 10^{3}$ to $35 \times 10^{6}$ (based on average velocity and diameter). Here, these experimental data are used to evaluate theories on the scaling in the overlap region. At sufficiently high Reynolds numbers, the mean velocity profile in the overlap region is found to be better represented by a log law than a power law. These results suggest a theory of complete similarity instead of incomplete similarity, contradicting the theories recently developed by Barenblatt et al.
\end{abstract}

PACS: 47.27.Jv, 47.27.Nz

\section{Introduction and Analysis}

A recent article in Science [1] highlights the controversy surrounding a new theory on the scaling of the overlap region in wall-bounded, turbulent shear flows. Barenblatt [2] was the first to propose this theory which predicts a power-law scaling for the intermediate or overlap region of the mean velocity profile. In the power law proposed by Barenblatt the multiplicative constant and the exponent depend on Reynolds number $[2,3,4,5]$. His analysis is based on the proposal of incomplete similarity and is at odds with traditional beliefs including the law-of-the-wall, the log law and 
Prandtl's Universal Law of Friction for Smooth Pipes [6] which are based on the proposal of complete similarity [7]. In the Science article, Chorin is quoted as saying, "The law of the wall was viewed as one of the few certainties in the difficult field of turbulence, and now it has been dethroned." He continues, "Generations of engineers who learned the law will have to abandon it." Before abandoning these conventional views, however, we believe a careful comparison with existing data is warranted.

Here, 26 mean velocity surveys taken in the Princeton University SuperPipe facility are used to compare theories on the scaling in the overlap region. These surveys span over three orders of magnitude in Reynolds numbers, $31 \times 10^{3}$ to $35 \times 10^{6}$. The Reynolds number is defined as

$$
R e=\frac{2 R \bar{U}}{\nu}
$$

where $\bar{U}$ is the average velocity, $R$ is the pipe radius and $\nu$ is the kinematic viscosity. Perhaps a more appropriate parameter to describe the characteristics of a wall-bounded, turbulent shear flow is the ratio of the global or inertial length scale to the viscous length. The viscous length is defined as $\nu / u_{\tau}$ where $u_{\tau}$ is the friction velocity defined as $\sqrt{\tau_{w} / \rho}$ where $\tau_{w}$ is the wall shear stress and $\rho$ is the fluid density. For a pipe, the ratio of length scales is given by

$$
R^{+}=\frac{R u_{\tau}}{\nu}
$$

The surveys presented here span a range of $R^{+}$from 850 to $530 \times 10^{3}$, a larger range of $R^{+}$than any other experiment in a single facility. We believe that the accuracy and $R^{+}$range of these measurements are sufficient to determine the scaling in the overlap region.

The experimental apparatus and technique, and the uncertainty analysis are described in [8], and they will only be summarized here. In this experiment, high Reynolds numbers were achieved by using compressed air (up to $187 \mathrm{~atm}$ ) as the working fluid, thereby decreasing the kinematic viscosity by over 2 orders of magnitude as compared to air at STP. The flow was shown to be fully-developed, symmetric and incompressible, and the pipe surface was shown to be smooth. The pipe diameter was $129 \mathrm{~mm}$, and the velocity profiles were measured using a $0.90 \mathrm{~mm}$ diameter round Pitot probe. For each survey, the velocity was measured at 52 wall-normal positions between $0.007 R$ and $1.5 R$ and the static wall pressure was measured at 20 taps equally spaced over 25 diameters. The static pressures were used to evaluate the static pressure gradient $d P / d x$ which in turn was used to evaluate

$u_{\tau}\left(u_{\tau}=\sqrt{\frac{d P}{d x} \frac{R}{2 \rho}}\right)$. Pitot probe corrections were applied according to [9] (see [8] for details). The overall uncertainty in the distance from the wall to the center of the Pitot probe was less than $\pm 0.05 \mathrm{~mm}( \pm 0.0008 R)$ which corresponds to an uncertainty in the normalized wall-normal position $\left(y^{+}=y u_{\tau} / \nu\right)$ of $\pm 11 \%$ at the wall and $\pm 0.99 \%$ at the centerline. Also, the Reynolds number had an uncertainty of $\pm 0.93 \%$, and the normalized velocity $\left(U^{+}=U / u_{\tau}\right)$ had an uncertainty of $\pm 0.57 \%$. 
In the conventional view, the mean velocity profile in turbulent pipe flow can be divided into two regions, a near-wall or inner region, and a core or outer region. In the inner region, the distance from the wall, the wall shear stress and the fluid properties are the only important parameters [10]. It follows that the functional dependence of the near-wall velocity profile is

$$
U=f_{0}\left[y, \tau_{w}, \rho, \mu\right]
$$

where $f_{0}$ expresses the functional relationship, $y$ is the distance from the wall and $\mu$ is the dynamic viscosity of the fluid. Dimensional analysis of equation 3 yields

$$
U^{+}=f_{1}\left[\frac{y u_{\tau}}{\nu}\right]=f_{1}\left[y^{+}\right]
$$

where $f_{1}$ expresses the dimensionless functional relationship. The region where equation 4 holds is known as the law-of-the-wall. If $f_{1}$ is independent of Reynolds number, complete similarity exists in this region. Otherwise, incomplete similarity exists.

In the outer region, the wall acts to retard the local velocity below the maximum velocity in a way which is independent of the viscosity, but dependent on the distance from the wall, the radius of the pipe and the velocity scale in the outer region [10]. The velocity profile in this region should scale according to

$$
U_{C L}-U=g_{0}\left[y, R, u_{0}\right]
$$

where $g_{0}$ expresses the functional relationship and $u_{0}$ is the velocity scale in the outer region. Dimensional analysis of equation 5 yields

$$
\frac{U_{C L}-U}{u_{0}}=g_{1}\left[\frac{y}{R}\right]
$$

where $g_{1}$ expresses the dimensionless functional relationship. The region where this functional relationship holds is known as the defect-law. If $g_{1}$ is independent of Reynolds number, then complete similarity exists in this region.

Between these two regions, there may exist an intermediate or overlap region where the distance from the wall is large compared to the inner length scale $\nu / u_{\tau}$, but small compared to the outer length scale $R$ (that is, $R^{+}$is large). In this article we confine our analysis to this intermediate or overlap region. For complete similarity, the velocity gradient in this region is independent of both the inner and outer length scale, and the scaling of the velocity profile can be shown to be logarithmic [10]. For incomplete similarity, the velocity gradient depends on one or both length scales, and the scaling of the velocity profile has been shown to be a power law using a number of different arguments $[2,11,8]$. 


\section{Results and Discussion}

In figure 1, we plot 13 mean velocity profiles taken in the SuperPipe facility normalized by inner scaling variables. From an analysis of all 26 velocity profiles, Zagarola \& Smits [12] concluded that for $y^{+}<0.1 R^{+}(y<0.1 R)$, the mean velocity profile is independent of Reynolds number, or equivalently, the outer length scale $R$. In figure 2 , all 26 velocity profiles are plotted for $y^{+}<0.1 R^{+}$ only, and no Reynolds number dependence is evident in this figure. Zagarola \& Smits also proposed that the mean velocity profile consists of two distinct regions: a power-law region for $50<y^{+}<500$ or $0.1 R^{+}$, and a log-law region for $500<y^{+}<0.1 R^{+}$. The power law is given by

$$
U^{+}=8.70\left(y^{+}\right)^{0.137},
$$

and the log law is given by

$$
U^{+}=\frac{1}{0.436} \ln y^{+}+6.13
$$

The numerical constants in equations 7 and 8 are empirical. Equations 7 and 8 are shown in figure 2 and appear to be in good agreement with the data.

For further comparison between equations 7 and 8 and the data, we plot the fractional differences in figures 3 and 4 . Here the fractional difference $\Delta U^{+} / U^{+}$is defined as

$$
\frac{\Delta U^{+}}{U^{+}}=1-\frac{U_{\text {predicted }}^{+}}{U_{\text {measured }}^{+}}
$$

where the predicted values are evaluated at the same $y^{+}$position as the measured values. When $\Delta U^{+} / U^{+}$is equal to zero, the measured and predicted values are equivalent. The fractional differences between equation 7 and the data are shown in figure 3 . The velocity data are within $\pm 0.78 \%$ ( $95 \%$ confidence interval) of the power law given by equation 7 , which is comparable to the uncertainty in $U^{+}( \pm 0.57 \%)$. This region is either part of the inner region which is well represented by a power law, or an overlap region exhibiting incomplete similarity (as defined in [12]). These results indicate that the power-law scaling exists in a discrete region between the inner region and outer region or logarithmic overlap region, depending on the magnitude of the Reynolds number, and the empirical constants in the power law do not depend on Reynolds number when expressed using inner scaling variables. Zagarola \& Smits argued that this region is an intermediate overlap region, but it is not the overlap region expected at very large Reynolds number. At very large Reynolds number, another overlap region is apparent, and the scaling in that region appears to be logarithmic. The fractional difference between the log law and the data is shown in figure 4 . The velocity data are within $\pm 0.68 \%$ ( $95 \%$ confidence interval) of the log law given by equation 8 , which is comparable to the uncertainty in $U^{+}( \pm 0.57 \%)$. 
In the theory proposed in [2], the multiplicative constant and the exponent in the power law are believed to be specific functions of Reynolds number. That is

$$
U^{+}=C[R e]\left(y^{+}\right)^{\gamma[R e]},
$$

where $C[R e]$ and $\gamma[R e]$ can be represented by an asymptotic expansion in $\varepsilon$ where $\varepsilon$ is given by

$$
\varepsilon=1 / \ln R e .
$$

The asymptotic expansions are

$$
C[R e]=C_{1} / \varepsilon+C_{2}+C_{3} \varepsilon+\cdots,
$$

and

$$
\gamma[R e]=\gamma_{1} \varepsilon+\gamma_{2} \varepsilon^{2}+\gamma_{3} \varepsilon^{3}+\cdots,
$$

where $C_{n}$ and $\gamma_{n}$ are empirically determined constants. This theory predicts an overlap region which exhibits incomplete similarity. (This is not the same type of incomplete similarity suggested by [12] (equation 7) where the empirical constants in the power law do not depend on Reynolds number and a log law is obtained at very large Reynolds numbers.) For the theory proposed in [2], the power-law scaling exists across most of the pipe radius and at all Reynolds numbers. Approximate limits for this region are $40<y^{+}<0.85 R^{+}$or $40 / R^{+}<y / R<0.85$. From an analysis of the data measured by Nikuradse [13], Barenblatt \& Prostokishin [3] determined that the values of $C_{1}, C_{2}$ and $\gamma_{1}$ were $0.577,2.50$, and 1.5 , respectively, and all other empirical constants could be set equal to zero with no loss of accuracy. This power law is given by

$$
U^{+}=(0.577 \ln R e+2.50)\left(y^{+}\right)^{1.5 / \ln R e} .
$$

For a complete description of this theory and the determination of the empirical constants see $[2,3,4]$ and $[5]$.

In figure 5, we show a comparison between equation 14 and the mean velocity data at 4 Reynolds numbers. The agreement is poor, particularly at the highest Reynolds number $\left(35 \times 10^{6}\right)$, but this may be expected since the constants in equation 14 were derived from Nikuradse's data which were limited to a maximum Reynolds number of $3.2 \times 10^{6}$ and are believed to be considerably less accurate than the data used here. For example, the uncertainty in the friction factors measured by Nikuradse was estimated to be over 3 times greater than the uncertainty in the friction factors evaluated from the data presented here $( \pm 1.1 \%)[8]$, and we expect the uncertainty in the $U^{+}$data to exhibit the same disparity. For a full evaluation of Nikuradse's data and experiment, see [8], and for an earlier assessment, see [14]. The relatively large uncertainty in Nikuradse's data casts considerable doubt on the values of the empirical constants determined in [3]. 
To further evaluate the theory given in [2], we determined the value of $C$ and $\gamma$ for each Reynolds number from the SuperPipe data using a least-squares approximation (no weighting) of equation 10 fitted to the data in the region $40<y^{+}<0.85 R^{+}$for all 26 velocity profiles. For each profile, there were typically 35 data points within the region of analysis, and the first three points nearest the wall were neglected due to the unacceptable uncertainty in their position. The Reynolds number dependence of $C$ and $\gamma$ are shown in figures 6 and 7 .

A least-squares approximation of equations 12 and 13 shows that at least two terms are required in the asymptotic expansion for $\gamma$ and $C$, rather than the one used by Barenblatt \& Prostokishin. Also, the values for $C_{1}$ and $C_{2}$ determined from our analysis are significantly different than the ones given in equation 14 which were determined by Barenblatt \& Prostokishin [3] using Nikuradse's data. Equation 10 can then be written as

$$
U^{+}=(0.7053 \ln R e+0.3055)\left(y^{+}\right)^{1.085 / \ln R e+6.535 / \ln R e^{2}}
$$

(where the constants were found using the SuperPipe data). Equation 15 is compared with the velocity data for 4 different Reynolds numbers in figure 8 . The agreement with the data is much improved for equation 15 than 14, but to compare this modified version of the power law proposed in [2] with the two-tier overlap region suggested in [12], we need to examine the fractional difference $\left(\Delta U^{+} / U^{+}\right)$between equation 15 and the data for all Reynolds numbers. Inspection of figure 9 reveals differences of $\pm 1 \%$ to $\pm 2.2 \%$ for a significant fraction of the data (more than $5 \%$ ) even though the uncertainty in the data is $\pm 0.57 \%$ ( $95 \%$ confidence interval). The maximum difference is near the wall at low Reynolds numbers (i.e. small $y^{+}$), and a definite pattern in $y^{+}$is evident.

To investigate any Reynolds number dependence, we plot the fractional difference at each Reynolds number on separate plots in figures 10 through 15. For all Reynolds numbers, for the outer region (say $y^{+}>0.2 R^{+}$) the fractional difference exhibits a characteristic "hook," and for the inner region (say $y^{+}<0.2 R^{+}$), a Reynolds number dependence is evident. If the scaling embodied in equation 15 is correct, then no patterns or Reynolds number dependencies would be observed. The only two Reynolds numbers at which no pattern is evident in the inner region are $140 \times 10^{3}$ and $180 \times 10^{3}$, where the multiplicative constant and the exponent are approximately equal to 8.70 and 0.137 , respectively (see figures 6 and 7), which are the same values determined in [12] for the power law with constant coefficients (equation 7).

The pattern in the inner region can be explained using a comparison between equations 7 and 8 with equation 15 . Here we define the fractional differences as

$$
\left.\frac{\Delta U^{+}}{U^{+}}\right|_{1}=1-\frac{U_{e q n 15}^{+}}{U_{e q n}^{+} 7},
$$


and

$$
\left.\frac{\Delta U^{+}}{U^{+}}\right|_{2}=1-\frac{U_{e q n 15}^{+}}{U_{\text {eqn } 8}^{+}} .
$$

The patterns observed for different Reynolds numbers in figures 16 and 17 are the same patterns observed when equation 15 is compared to the measured data (only $y^{+}$values common to the region of validity of both equations are shown). We believe that this provides strong support for the scaling proposed in [8] and [12] (equations 7 and 8) better represents this mean velocity data than the scaling proposed in $[2,3,4,5]$.

\section{Conclusions}

The mean flow data measured in the Princeton University SuperPipe support a logarithmic overlap region for $500<y^{+}<0.1 R^{+}$. There also appears to be a power-law region for $50<y^{+}<500$ or $0.1 R^{+}$which could be regarded as part of the inner region, or perhaps it is a second overlap region exhibiting incomplete similarity as suggested by Zagarola et al. [8, 12]. A logarithmic overlap region at sufficiently high Reynolds numbers suggests a theory of complete similarity instead of incomplete similarity contradicting the theories recently developed by Barenblatt et al. [2, 3, 4, 5]. Although this comparison was specifically between the scaling proposed by Zagarola et al. and Barenblatt et $a l$., the results should hold for any power-law representation of the mean velocity profile which has approximate limits defined by $40<y^{+}<0.85 R^{+}$or $40 / R^{+}<y / R<0.85$.

\section{Acknowledgments}

The support of ARPA/ONR under Grant N00014-92-J-1796 is gratefully acknowledged. 


\section{References}

[1] B. Cipra. A new theory of turbulence causes a stir among experts. Science, 272, May 1996.

[2] G.I. Barenblatt. Scaling laws for fully developed turbulent shear flows. Part 1. Basic hypotheses and analysis. Journal of Fluid Mechanics, 248:513-520, 1993.

[3] G.I. Barenblatt and V.M. Prostokishin. Scaling laws for fully developed turbulent shear flows. Part 2. Processing of experimental data. Journal of Fluid Mechanics, 248, 1993.

[4] G.I. Barenblatt and N. Goldenfeld. Does fully developed turbulence exist? Reynolds number independence versus asymptotic covariance. Physics of Fluids, 7(12), 1995.

[5] G.I. Barenblatt and A.J. Chorin. Scaling laws and zero viscosity limits for wall-bounded shear flows and for local structure in developed turbulence. Center for Pure and Applied Mathematics University of California at Berkeley Internal Report, PAM-678, May 1996.

[6] H. Schlichting. Boundary-Layer Theory. McGraw-Hill, 7th edition, 1979.

[7] M.V. Zagarola and A.J. Smits Reynolds number dependence of the mean flow in a circular pipe. AIAA Paper 97-0649, 1997.

[8] M.V. Zagarola. Mean flow scaling in turbulent pipe flow. Ph.D. Thesis, Princeton University, 1996.

[9] S.H. Chu. Pressure probes for fluid measurements. Progress in Aerospace Sciences, 16:147-223, 1975.

[10] H. Tennekes and J.L. Lumley. A First Course in Turbulence. MIT Press, 1972.

[11] W.K. George, P. Knecht, and L. Castillo. The zero-pressure gradient turbulent boundary layer revisited. In Proceedings of the Thirteenth Biennial Symposium on Turbulence, University of Missouri-Rolla, 1992.

[12] M.V. Zagarola and A.J. Smits. Scaling of the mean velocity profile for turbulent pipe flow. Physics Review Letters, 78(2), 1997.

[13] J. Nikuradse. Gesetzmäßigkeit der turbulenten Stromüng in glatten Rohren. Forsch. Arb. Ing.-Wes. No. 356, 1932. English translation NACA TT F-10, 359.

[14] J.O. Hinze. Turbulent pipe flow. In The Mechanics of Turbulence. International Symposium of the National Scientific Research Center, Marseille, Gordon and Breach, 1961. 


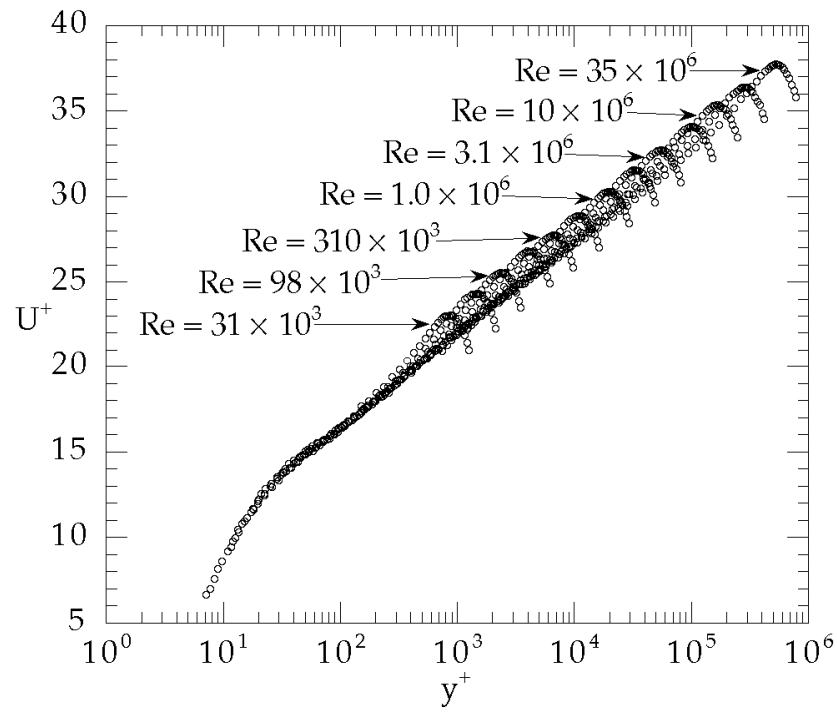

Figure 1: Plot of the velocity profiles normalized using inner scaling variables for 13 different Reynolds number between $31 \times 10^{3}$ and $35 \times 10^{6}$.

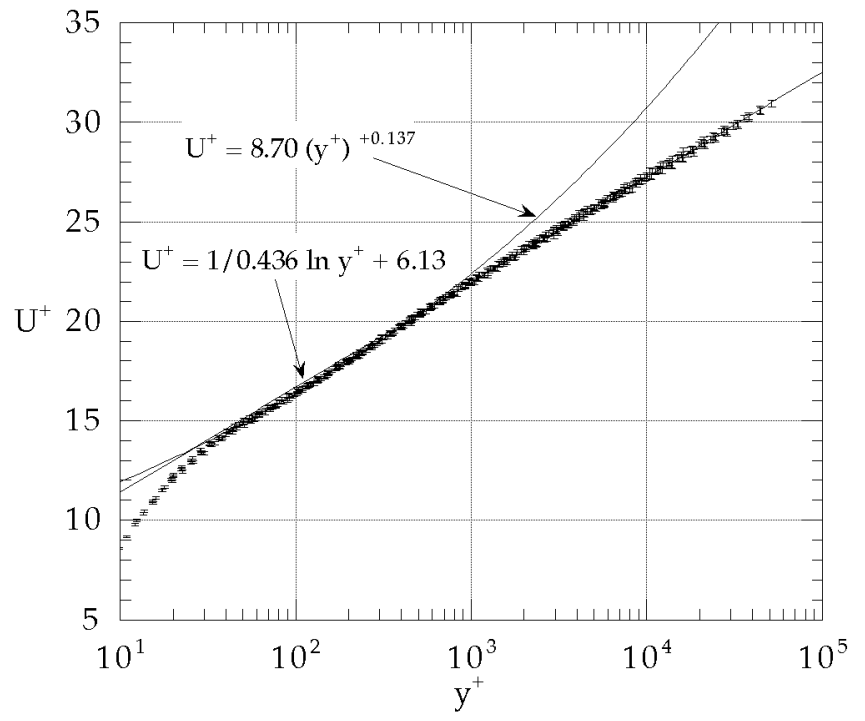

Figure 2: Plot of the velocity profiles within $0.1 \mathrm{R}$ of the wall normalized using inner scaling variables for 26 different Reynolds number between $31 \times 10^{3}$ and $35 \times 10^{6}$. The error bars represent an uncertainty in $U^{+}$of $\pm 0.57 \%$. 


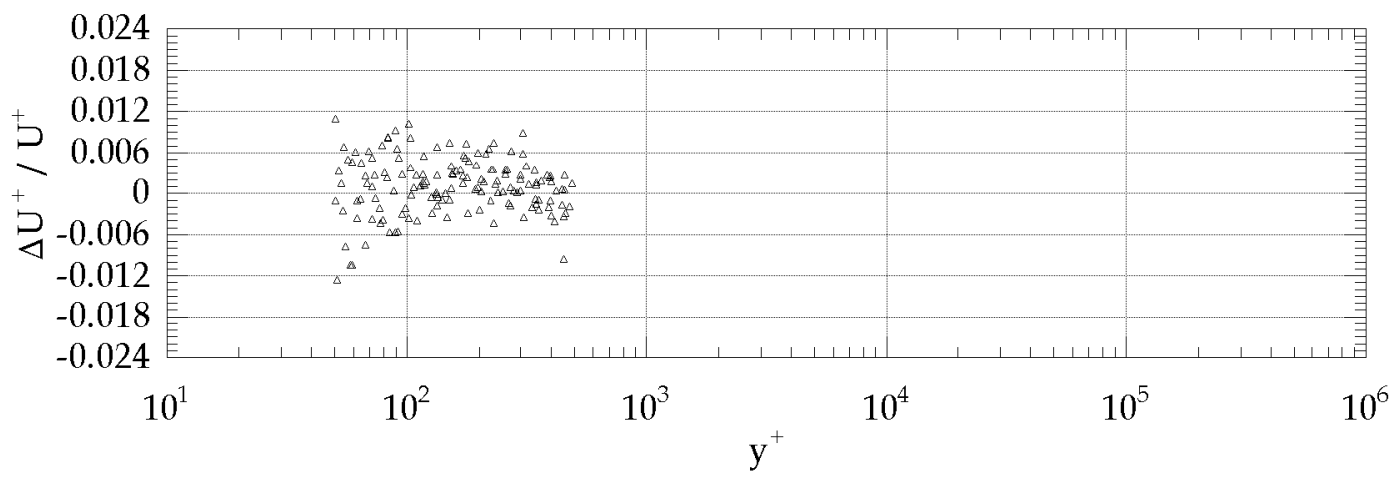

Figure 3: Plot of the fractional difference between the power law given in equation 6 and the velocity profile data. Only data between $50<y^{+}<500$ are shown.

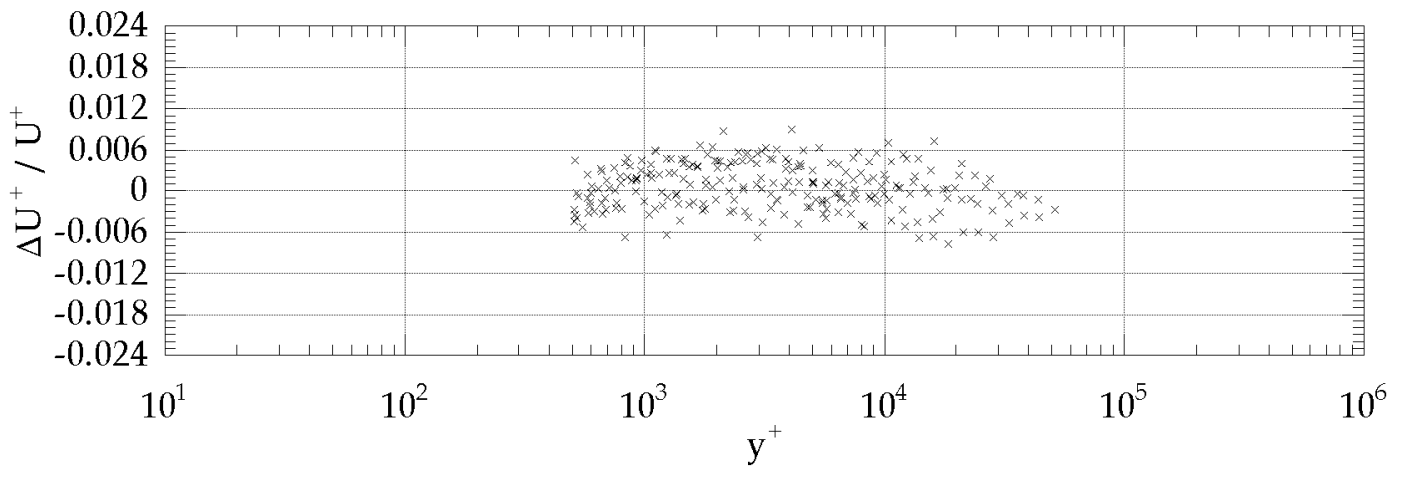

Figure 4: Plot of the fractional difference between the log law given in equation 7 and the velocity profile data. Only data between $500<y^{+}<0.1 R^{+}$are shown. 


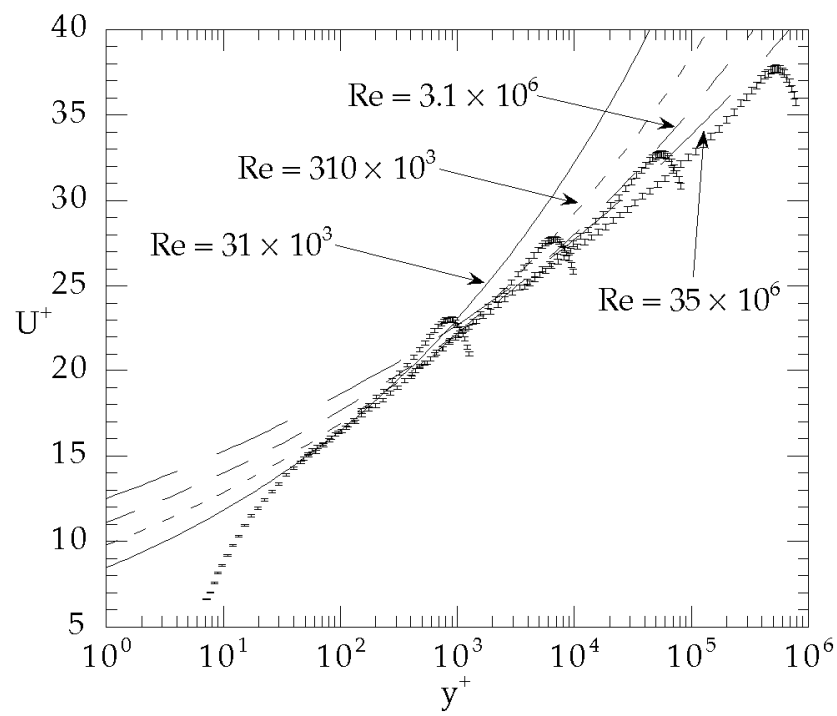

Figure 5: Comparison between the mean velocity profiles and equation 14 for 4 different Reynolds number between $31 \times 10^{3}$ and $35 \times 10^{6}$. The error bars represent an uncertainty in $U^{+}$of $\pm 0.57 \%$.

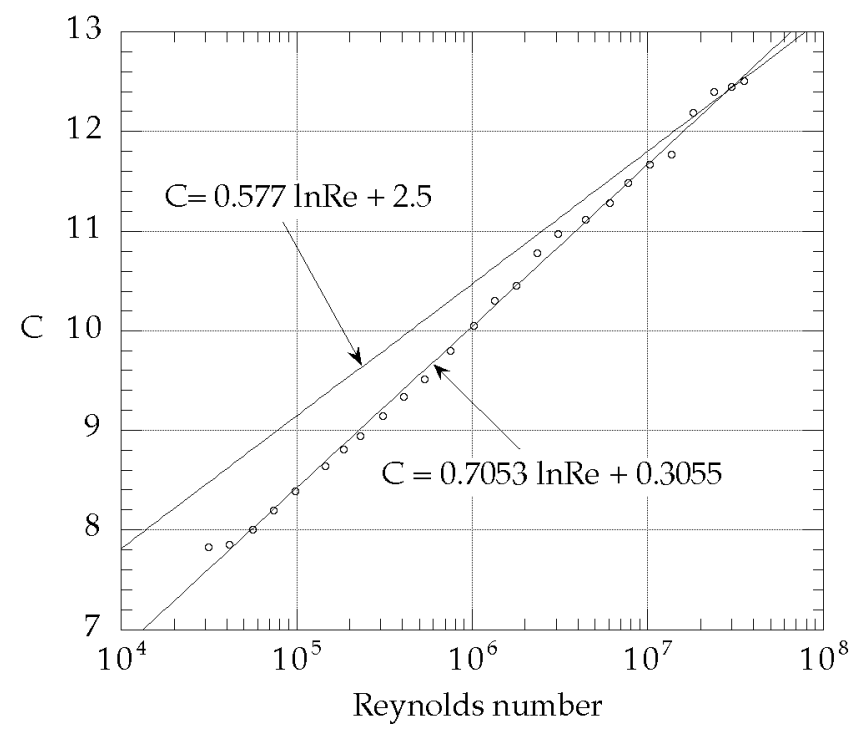

Figure 6: Plot of the multiplicative constant in the power law given by equation 10 for 26 different Reynolds number between $31 \times 10^{3}$ and $35 \times 10^{6}$. 


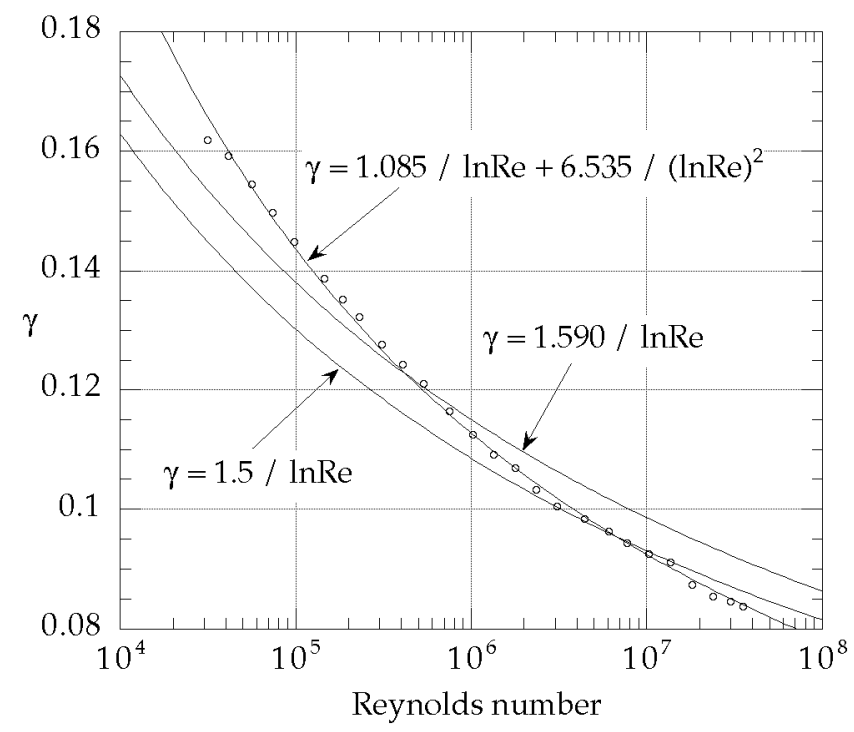

Figure 7: Plot of the exponent in the power law given by equation 10 for 26 different Reynolds number between $31 \times 10^{3}$ and $35 \times 10^{6}$.

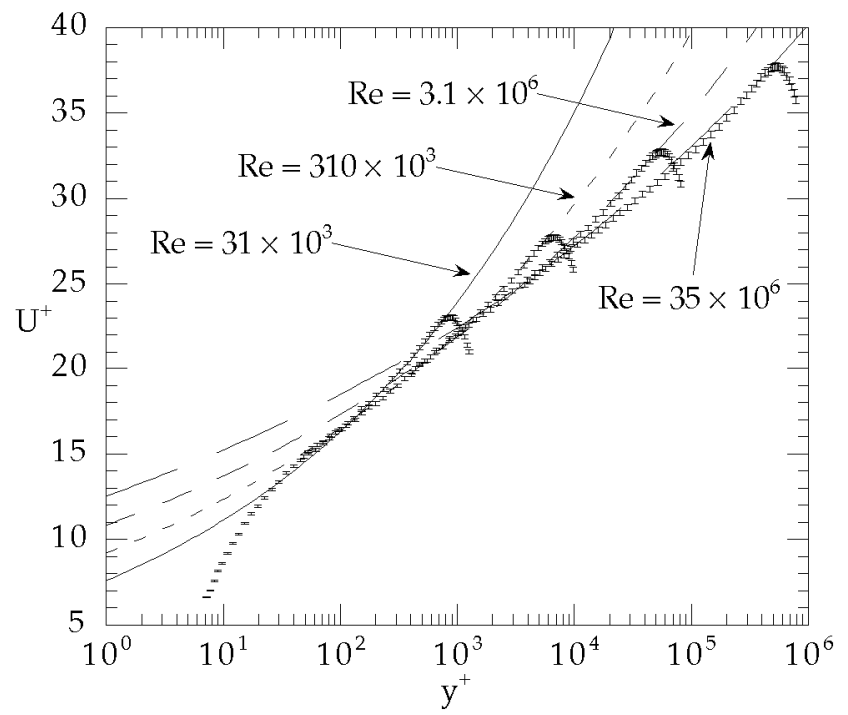

Figure 8: Comparison between the mean velocity profiles and equation 15 for 4 different Reynolds number between $31 \times 10^{3}$ and $35 \times 10^{6}$. The error bars represent an uncertainty in $U^{+}$of $\pm 0.57 \%$. 


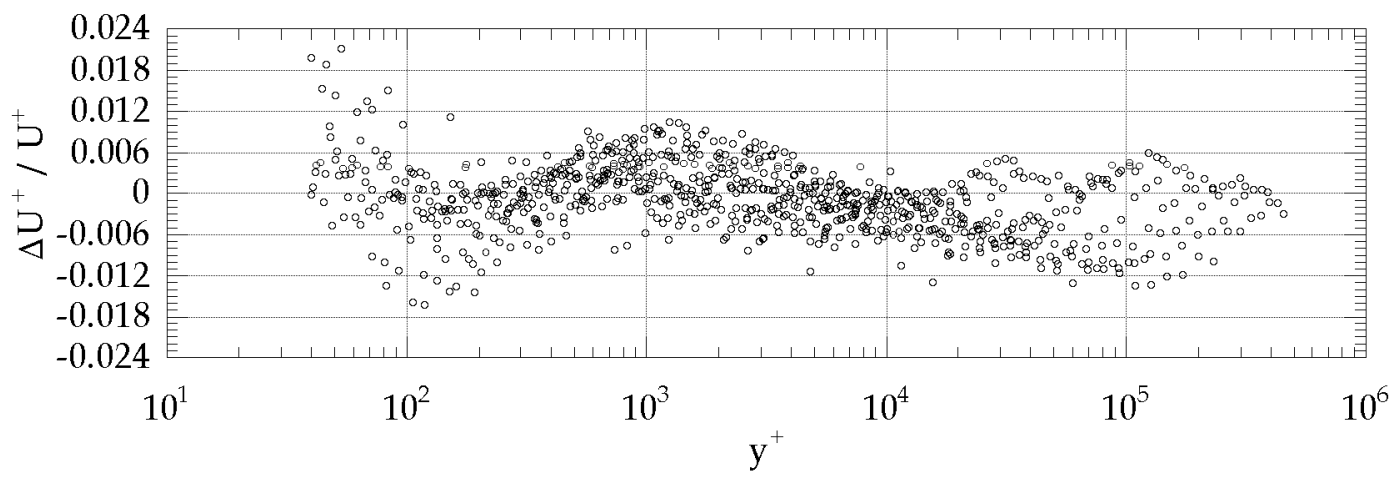

Figure 9: Plot of the fractional difference between the power law given by equation 15 and the velocity profile data. Only data between $40<y^{+}<0.85 R^{+}$are shown for each Reynolds number.

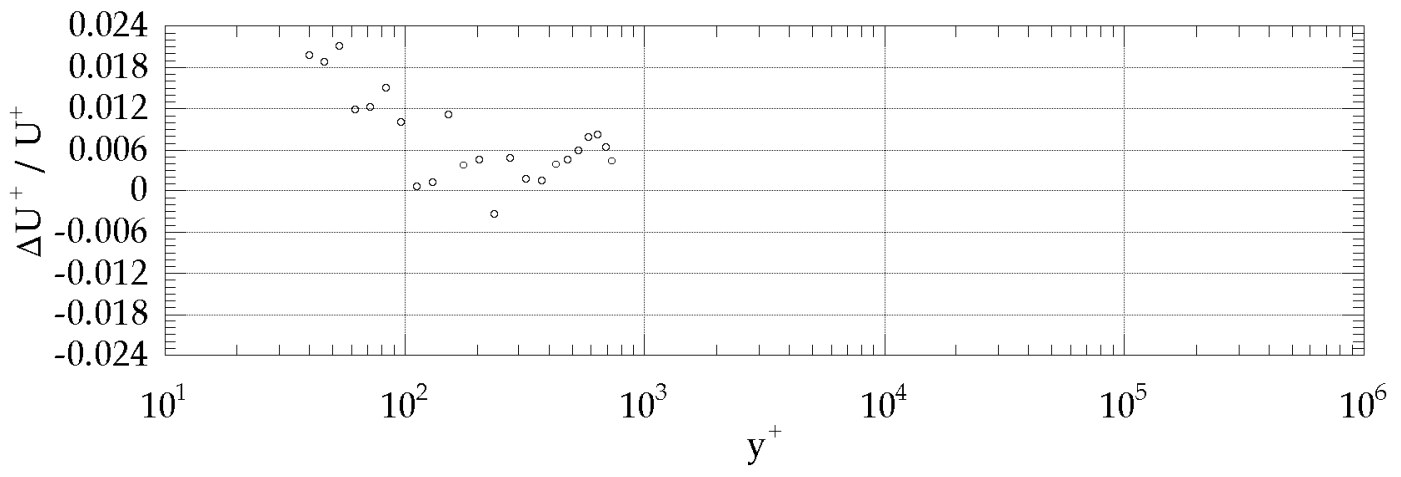

Figure 10: Plot of the fractional difference between the power law given by equation 15 and the velocity profile data for $R e=31 \times 10^{3}$. Only data between $40<y^{+}<0.85 R^{+}$are shown. 

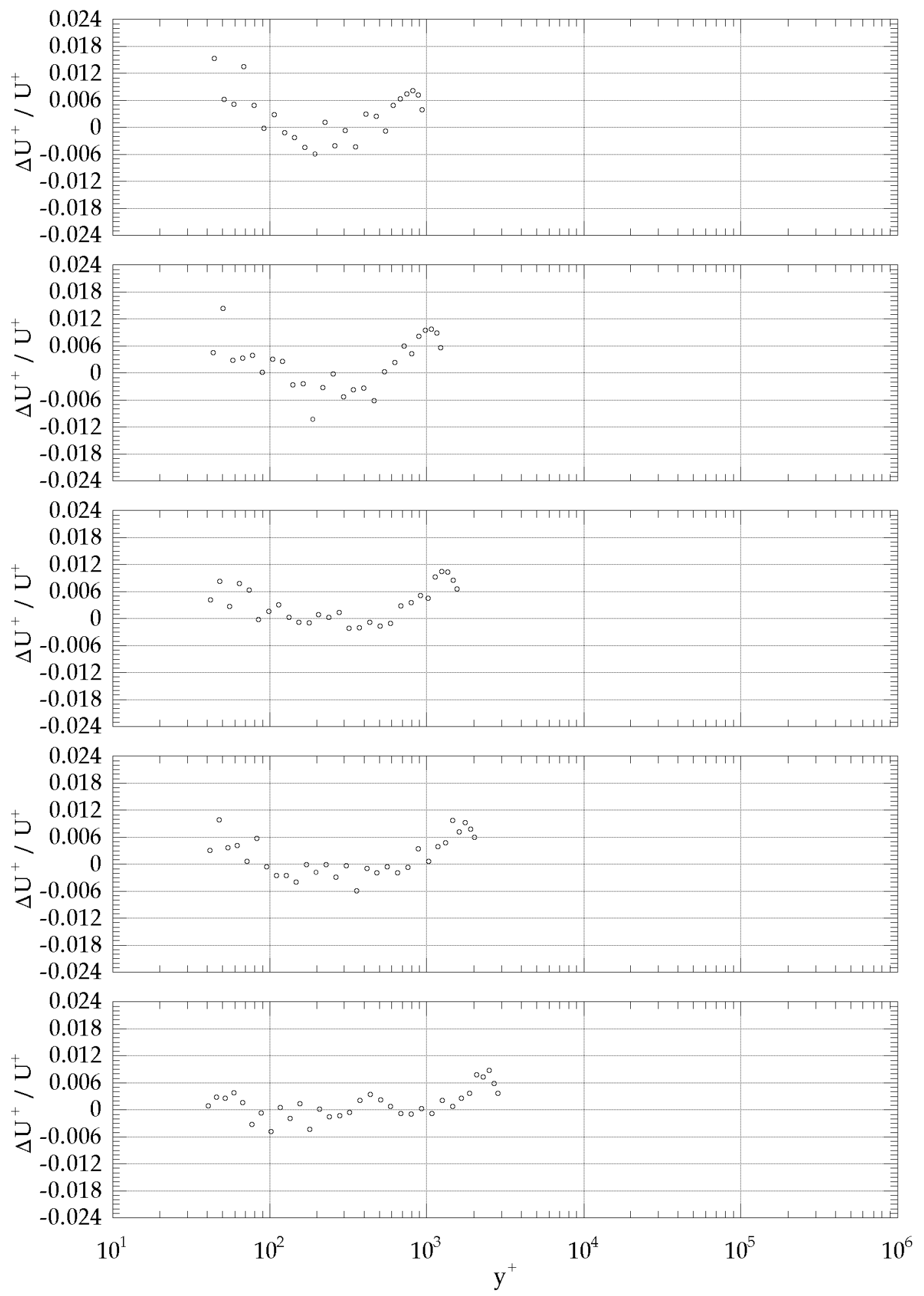

Figure 11: Plot of the fractional difference between the power law given by equation 15 and the velocity profile data for different Reynolds numbers. Only data between $40<y^{+}<0.85 R^{+}$are shown. From top to bottom, the graphs correspond to $R e=41 \times 10^{3}, 56 \times 10^{3}, 74 \times 10^{3}, 98 \times 10^{3}$, and $140 \times 10^{3}$. 

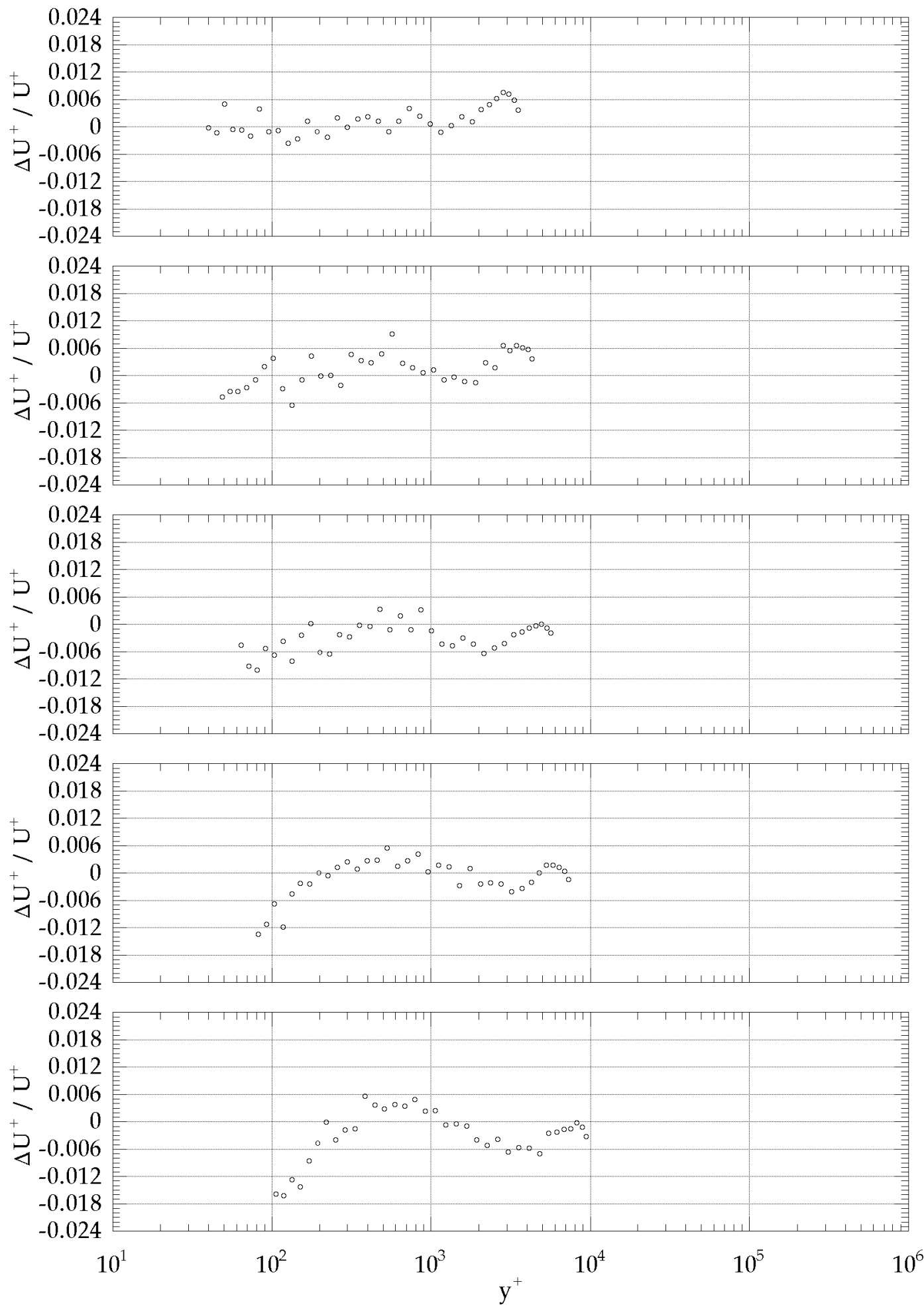

Figure 12: Plot of the fractional difference between the power law given by equation 15 and the velocity profile data for different Reynolds numbers. Only data between $40<y^{+}<0.85 R^{+}$are shown. From top to bottom, the graphs correspond to $R e=180 \times 10^{3}, 230 \times 10^{3}, 310 \times 10^{3}, 410 \times 10^{3}$, and $540 \times 10^{3}$. 

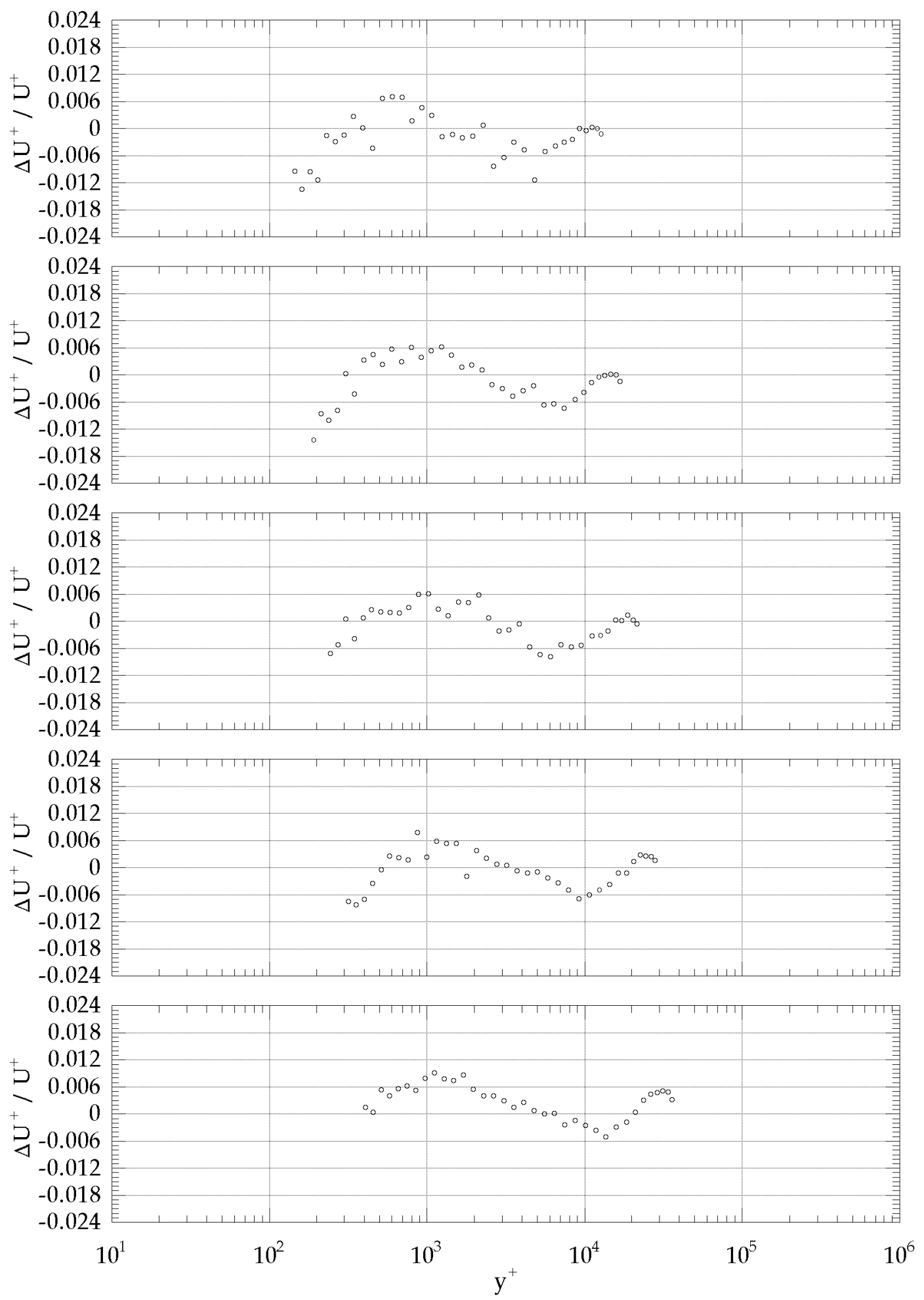

Figure 13: Plot of the fractional difference between the power law given by equation 15 and the velocity profile data for different Reynolds numbers. Only data between $40<y^{+}<0.85 R^{+}$are shown. From top to bottom, the graphs correspond to $R e=750 \times 10^{3}, 1.0 \times 10^{6}, 1.3 \times 10^{6}, 1.8 \times 10^{6}$, and $2.3 \times 10^{6}$. 

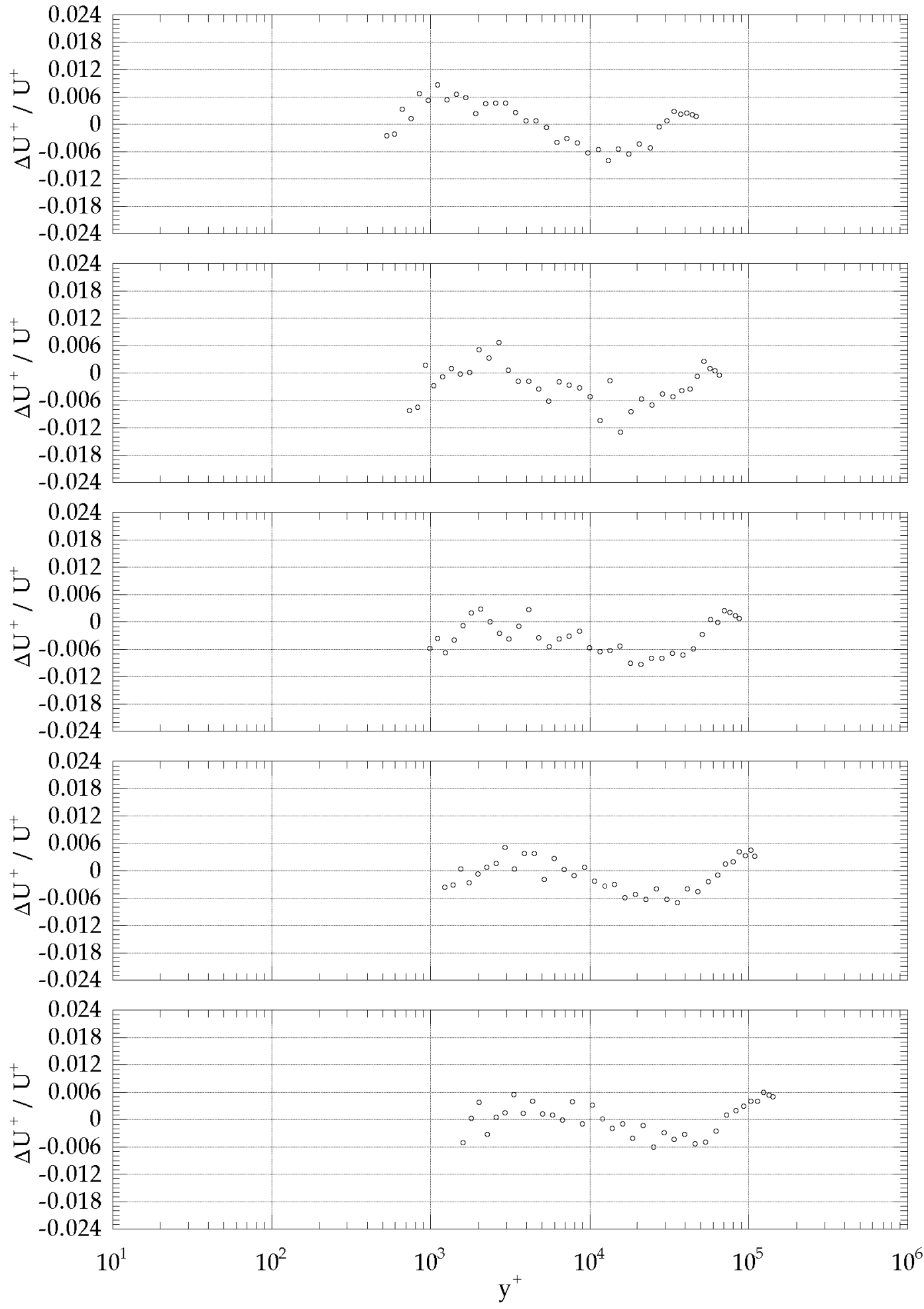

Figure 14: Plot of the fractional difference between the power law given by equation 15 and the velocity profile data for different Reynolds numbers. Only data between $40<y^{+}<0.85 R^{+}$are shown. From top to bottom, the graphs correspond to $R e=3.1 \times 10^{6}, 4.4 \times 10^{6}, 6.1 \times 10^{6}, 7.7 \times 10^{6}$, and $10 \times 10^{6}$. 

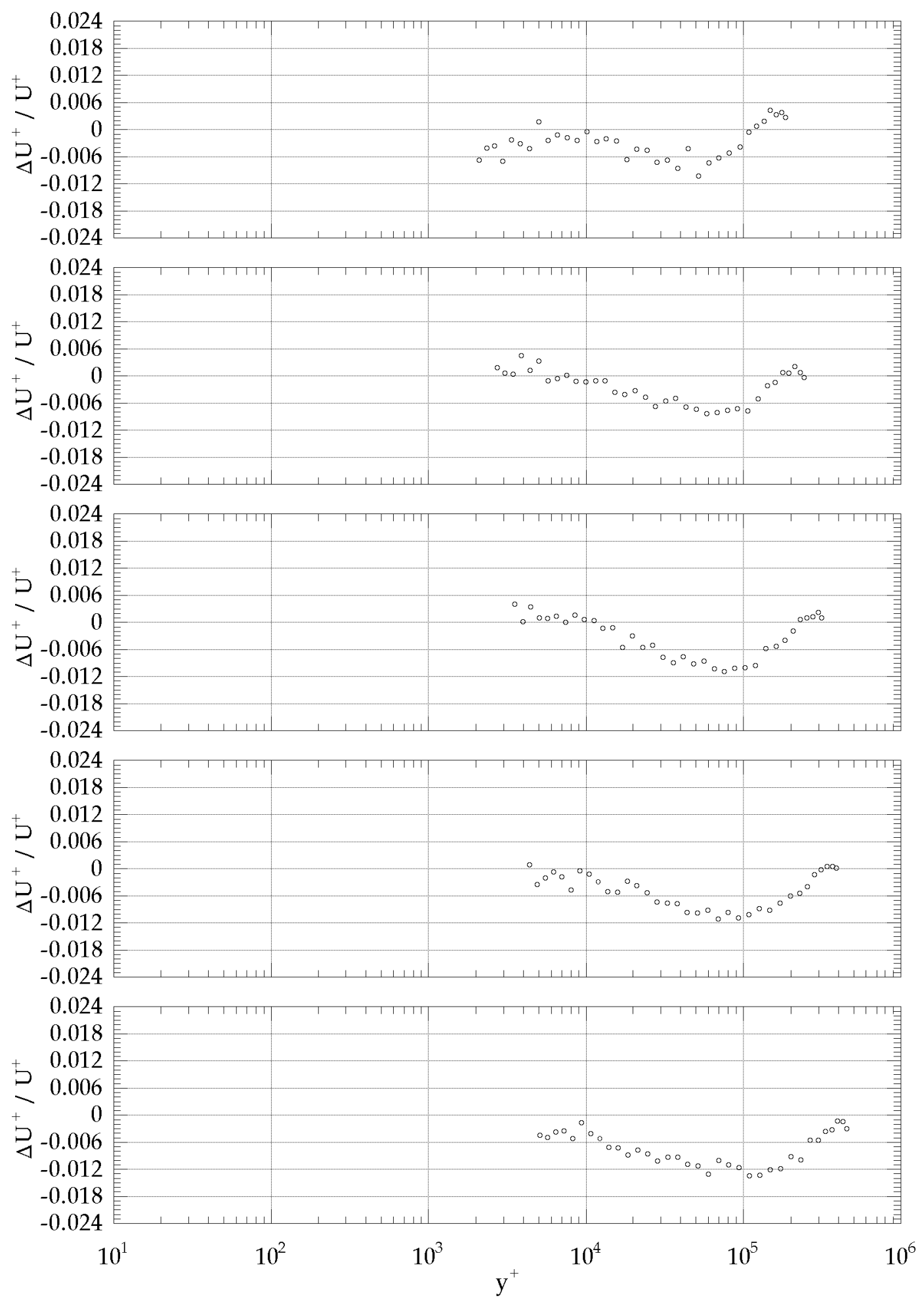

Figure 15: Plot of the fractional difference between the power law given by equation 15 and the velocity profile data for different Reynolds numbers. Only data between $40<y^{+}<0.85 R^{+}$are shown. From top to bottom, the graphs correspond to $R e=14 \times 10^{6}, 18 \times 10^{6}, 24 \times 10^{6}, 31 \times 10^{6}$, and $35 \times 10^{6}$. 


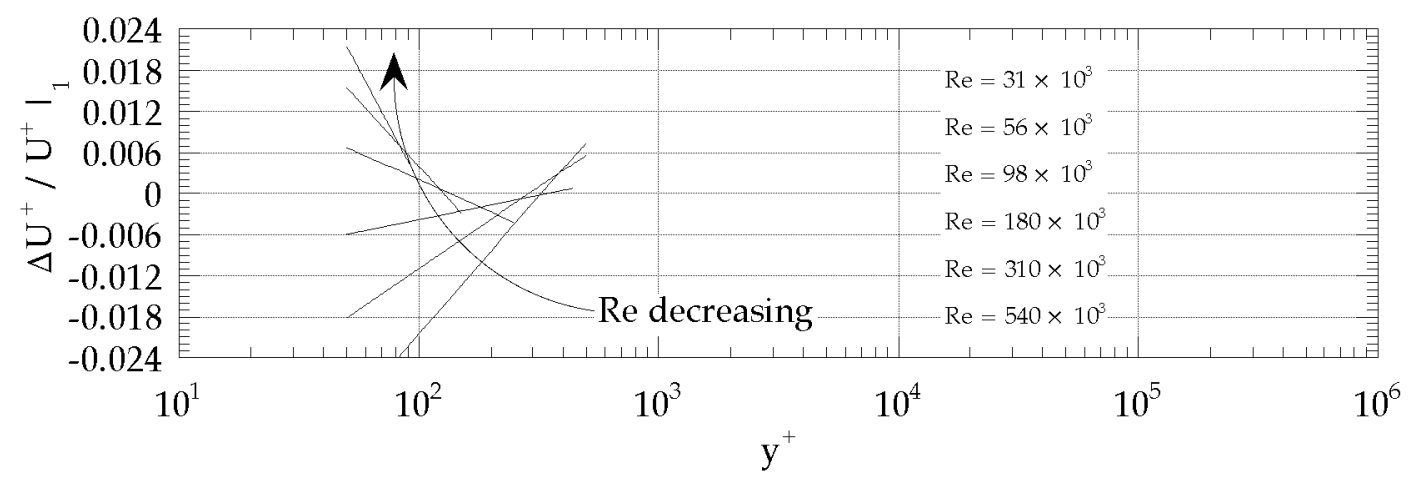

Figure 16: Plot of the fractional difference between the power law given by equation 15 and the power law given by equation 7 . Only $y^{+}$values common to the region of validity of both equations are shown.

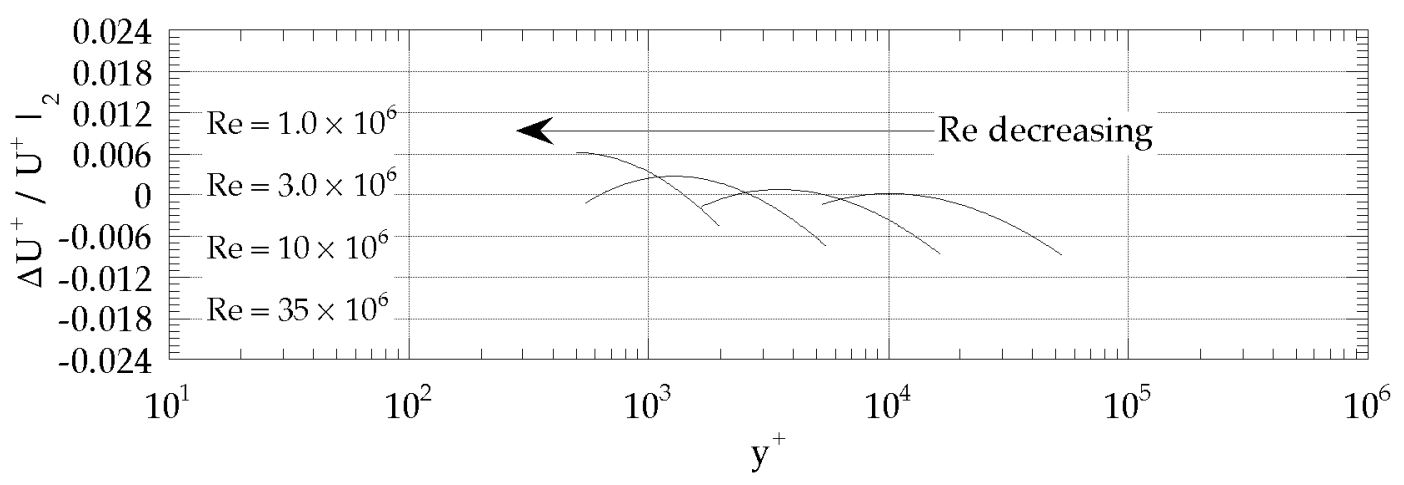

Figure 17: Plot of the fractional difference between the power law given by equation 15 and the log law given by equation 8 . Only $y^{+}$values common to the region of validity of both equations are shown. 